\title{
Association of cardiac baroreflex sensitivity with blood pressure transients: influence of sex and menopausal status
}

\author{
Jill N. Barnes ${ }^{1}{ }^{*}$, Luke J. Matzek ${ }^{1}$, Nisha Charkoudian ${ }^{2}$, Michael J. Joyner ${ }^{1}$, Timothy B. Curry ${ }^{1}$ and \\ Emma C. Hart ${ }^{1}$
}

${ }^{1}$ Human Integrative Physiology Laboratory, Department of Anesthesiology, Mayo Clinic, Rochester, MN, USA

2 Thermal and Mountain Medicine Division, U.S. Army Research Institute of Environmental Medicine, Natick, MA, USA

Edited by:

Elisabeth Lambert, Baker IDI Heart and Diabetes Institute, Australia

\section{Reviewed by:}

J. Kevin Shoemaker, The University of

Western Ontario, Canada

Qi Fu, The University of Texas

Southwestern Medical Center at

Dallas, USA

*Correspondence:

Jill N. Barnes, Human Integrative Physiology Laboratory, Department of Anesthesiology, Mayo Clinic, 200 1st Street SouthWest, SMH, Joseph 4-184, Rochester, MN 55905, USA. e-mail: barnes.jill@mayo.edu
The magnitude of decrease in blood pressure (BP) during a vasoactive drug bolus may be associated with the calculated baroreflex sensitivity (BRS). The purpose of the present study was to evaluate whether sympathetic and/or cardiac BRS relates to the extent of change in BP and whether this was altered by sex hormones. Fifty-one young women ( $27 \pm 1$ years), 14 older women ( $58 \pm 1$ years), and 36 young men ( $27 \pm 1$ years) were studied. Heart rate, BP, and muscle sympathetic nerve activity (MSNA) were monitored. Sympathetic BRS was analyzed using the slope of the MSNA-diastolic blood pressure (DBP) relationship and cardiac BRS was analyzed using the $R-R$ interval-systolic blood pressure (SBP) relationship. Young women and men had similar mean arterial pressures (MAP, $91 \pm 1$ vs. $90 \pm 1 \mathrm{mmHg}$ ), cardiac BRS (19 $\pm 1 \mathrm{vs.} 21 \pm 2 \mathrm{~ms} / \mathrm{mmHg}$ ), and sympathetic BRS $(-6 \pm 1$ vs. $-7 \pm 1 \mathrm{AU} / \mathrm{beat} / \mathrm{mmHg})$, respectively. Older women had higher MAP $(104 \pm 4 \mathrm{mmHg}$, $p<0.05)$ and lower cardiac BRS $(7 \pm 1 \mathrm{~ms} / \mathrm{mmHg}, p<0.05)$, but similar sympathetic BRS $(-8 \pm 1 \mathrm{AU} / \mathrm{beat} / \mathrm{mmHg})$. There was no association between BP transients with either cardiac or sympathetic BRS in young women. In the older women, the drop in SBP, DBP, and MAP were associated with cardiac BRS $(r=0.60, r=0.59$, and $r=0.70$, respectively; $p<0.05)$, but not sympathetic BRS. The decrease in SBP was positively related to cardiac BRS in young men $(r=0.41 ; p<0.05)$. However, there was no relationship between the decrease in BP and sympathetic BRS. This indicates that older women and young men with low cardiac BRS have larger transients in BP during nitroprusside. This suggests a more prominent role for cardiac (as opposed to sympathetic) BRS in responding to acute $\mathrm{BP}$ changes in young men and older women. The fact that these relationships do not exist in young women suggest that the female sex hormones influence baroreflex responses.

Keywords: cardiovagal, sympathetic nerve activity, aging, blood pressure regulation

\section{INTRODUCTION}

The arterial baroreflex is essential in buffering acute hypertensive and hypotensive episodes in order to maintain blood pressure (BP) at normal levels during daily activities and changes in posture. Arterial baroreflex sensitivity (BRS) can be assessed during vasoactive drug infusions, using neck suction/neck pressure or other maneuvers to acutely alter $\mathrm{BP}$. The response of the cardiac arm of the reflex is evaluated by measuring the changes in $\mathrm{R}-\mathrm{R}$ interval (RRI) or heart rate (HR); whereas muscle sympathetic nerve activity (MSNA) responses are used to quantify the sympathetic arm during the transient changes in BP. Recent studies have shown a dissociation between cardiac and sympathetic BRS in young adults (O'Leary et al., 2005; Dutoit et al., 2010), emphasizing that it should not be assumed that the sensitivity of one arm of the reflex is reflective of the sensitivity of the other.

By definition, a person with low BRS has a smaller efferent autonomic response for a given change in pressure. Consequently, an individual with less baroreflex responsiveness should have less ability to buffer decreases in BP in response to hypotensive stimuli. The cardiac or sympathetic component of the baroreflex may defend against a drop in BP. However, this relationship has never been directly assessed. Therefore, we sought to test the hypothesis that the transient drop in BP during a modified Oxford baroreflex test was associated with the calculated cardiac and sympathetic BRS.

Emerging evidence suggests the presence of sex-related differences in the autonomic regulation of arterial BP in humans (Shoemaker et al., 2001; Christou et al., 2005; Hart et al., 2009a). Furthermore, it is well known that orthostatic intolerance and other hypotensive disorders are more prevalent in young women, compared to men of the same age (Convertino, 1998; \#2400). However, there is conflicting evidence as to whether sex-specific differences in baroreflex control of BP exist (Tank et al., 2005; Johnson et al., 2011). Accordingly, we examined whether there were potential sex differences in the association between the acute decrease in BP following a vasoactive drug infusion with cardiac 
or sympathetic BRS in young women compared to young men. Because any sex-related differences may reflect the influence of female hormones, we also investigated these relationships in older postmenopausal women.

\section{MATERIALS AND METHODS SUBJECTS}

Subject participants were recruited from Mayo Clinic and the surrounding southeastern Minnesota. Healthy subjects included 36 young men ( $27 \pm 1$ years), 51 young women ( $27 \pm 1$ years), and 14 postmenopausal women ( $58 \pm 2$ years). Subjects who were pregnant, had chronic diseases, or took drugs known to affect endocrine, cardiovascular, autonomic function, or were taking hormone replacement therapy were excluded. Young women taking oral contraceptives were allowed to participate in the study. All subjects were given informed consent and screened for height, weight, and overall physical health. All procedures had ethical approval from the Institutional Review Board at the Mayo Clinic and were performed according to the Declaration of Helsinki including written informed consent.

\section{HEMODYNAMIC MEASUREMENTS}

All studies were performed in a clinical research laboratory in the Mayo Clinic Center for Translational Science Activities. Studies were conducted in a climate-controlled room $\left(22-24^{\circ} \mathrm{C}\right)$, with subjects resting in a supine or semi-recumbent position and care taken to minimize distractions. Subjects arrived at the laboratory after an overnight fast and abstinence from alcohol and caffeine for $24 \mathrm{~h}$ prior to the study. Throughout the experiment, HR was measured from a 3-lead ECG. Brachial BP was measured from a $5-\mathrm{cm}$ catheter, which was placed into the brachial artery under aseptic conditions using ultrasound guidance and local anesthesia (2\% lidocaine). In addition, an intravenous catheter was placed into a hand vein for drug administration.

\section{MUSCLE SYMPATHETIC NERVE ACTIVITY}

Microneurography was performed using a tungsten microelectrode placed into the peroneal nerve, posterior to the fibular head as described in previous studies (Sundlof and Wallin, 1977; Wallin et al., 2010). The signal was amplified 80,000-fold, bandpass filtered $(700-2000 \mathrm{~Hz})$, rectified and integrated (resistancecapacitance integrator circuit, time constant $0.1 \mathrm{~s}$ ) by a nervetraffic analyzer. Data were recorded at $250 \mathrm{~Hz}$ using a computer data acquisition system (WinDaq, DATAQ Instruments, Akron, $\mathrm{OH}$, USA) and stored for off-line analysis. Sympathetic bursts in the integrated neurogram were identified using a custommanufactured automated analysis program (Kienbaum et al., 2001). Burst identification was then corrected by a single observer. The program then compensated for baroreflex latency and associated each sympathetic burst with the appropriate cardiac cycle. MSNA was quantified as bursts per minute (burst frequency, BF) and bursts per 100 heartbeats (burst incidence, BI).

\section{BAROREFLEX SENSITIVITY}

The modified Oxford technique was used to evaluate BRS as previously described (Charkoudian et al., 2004). Briefly, sodium nitroprusside $(100 \mu \mathrm{g})$ was injected through a venous catheter inserted into an antecubital vein in the dominant arm, which was followed by phenylephrine $(150 \mu \mathrm{g}) 1 \mathrm{~min}$ later. Data were collected for an additional $2 \mathrm{~min}$.

\section{Cardiac BRS}

Cardiac BRS was assessed by using the linear relationship between RRI and systolic blood pressure (SBP) during changes in pressure during the modified Oxford technique (Charkoudian et al., 2004). Values for RRI were pooled over $2-\mathrm{mmHg}$ ranges. To be acceptable for inclusion in our analysis, each regression line had to contain 12-15 points and have a correlation coefficient greater than $r=0.80$. We focused on RRI responses to BP changes as these have been shown to be directly related to efferent vagal activity to the heart (Parker et al., 1984).

\section{Sympathetic BRS}

Sympathetic BRS was assessed by using the relationship between MSNA and diastolic Blood Pressure (DBP). Using a custom computer software program (Halliwill, 2000), the slope of the linear portion of the relationship between MSNA (total activity) and DBP was used as an index of BRS. For this analysis, MSNA values were combined into $3 \mathrm{mmHg}$ bins. As described previously, this "binning" reduces the statistical impact of the non-baroreflex beat-to-beat variability in MSNA (Dutoit et al., 2010). A window of nerve activity that was $1.0 \mathrm{~s}$ in length and synchronized by the R wave of the ECG was signal averaged. The window was time shifted to account for the latency between $\mathrm{R}$ waves and sympathetic bursts. The duration of the shift was varied as needed from subject to subject. Any cardiac cycle not followed by a burst was assigned a total integrated activity of 0 . The sensitivity of the baroreflex measured by the modified Oxford method was defined as the slope of the linear regression between MSNA and the means of the DBP bins.

\section{BLOOD PRESSURE TRANSIENTS}

Mean arterial pressure (MAP) was calculated using the equation $\mathrm{MAP}=\mathrm{DBP}+1 / 3(\mathrm{PP})$ and $\mathrm{PP}=\mathrm{SBP}-\mathrm{DBP}$. A customized Microsoft excel worksheet calculated the beat-by-beat BP (systolic, diastolic, mean, and pulse) from WinDaq data acquisition system. Five minutes of average baseline BP and the nadir pressure (the lowest $\mathrm{BP}$ in response to vasoactive nitroprusside) were calculated. The change in BP was the nadir pressures minus the baseline. In other words, the decreasing pressure was calculated by subtracting the lowest detected pressure during infusion of nitroprusside from a mean of the baseline pressures.

\section{STATISTICS}

Descriptive statistics were used for the analysis of demographic data. Group differences were analyzed using a one-way ANOVA. Spearman's rho correlations were used to determine the association between BP changes and cardiac or sympathetic BRS. Separate baroreflex curves to increasing or decreasing pressures were not calculated because we would not have enough data points to perform linear regression analysis, particularly in the young subjects. A significance level of $p<0.05$ was set $a$ priori to determine statistical significance. 
Table 1 | Subject demographics.

\begin{tabular}{llll}
\hline & Young women & Young men & Older women \\
\hline$n$ & 51 & 36 & 14 \\
Age, years & $27 \pm 1$ & $27 \pm 1$ & $58 \pm 2$ \\
BMI, kg/m² & $23.0 \pm 0.3$ & $24.4 \pm 0.4$ & $24.4 \pm 0.6$ \\
$\begin{array}{l}\text { Heart rate, bpm } \\
\text { Systolic blood }\end{array}$ & $62 \pm 1$ & $58 \pm 1$ & $62 \pm 2$ \\
pressure, mmHg & $127 \pm 2$ & $130 \pm 2$ & $146 \pm 6^{*}$ \\
$\begin{array}{l}\text { Diastolic blood pres- } \\
\text { sure, mmHg }\end{array}$ & $71 \pm 1$ & $71 \pm 1$ & $75 \pm 2$ \\
$\begin{array}{l}\text { Mean arterial pressure, } \\
\text { mmHg }\end{array}$ & $91 \pm 1$ & $90 \pm 1$ & $104 \pm 4^{*}$ \\
$\begin{array}{l}\text { Pulse pressure, mmHg } \\
\text { MSNA, bursts/100 hb }\end{array}$ & $56 \pm 2$ & $59 \pm 2$ & $71 \pm 5^{*}$ \\
\end{tabular}

Data are mean \pm standard error. MSNA, muscle sympathetic nerve activity. ${ }^{*} p<0.05$ vs. young women and young men.

Table 2 | BRS and change in blood pressure during the modified Oxford.

\begin{tabular}{llll}
\hline & Young women & Young men & $\begin{array}{l}\text { Older } \\
\text { women }\end{array}$ \\
\hline Cardiac BRS, ms/mmHg & $20 \pm 1.7$ & $21 \pm 2.1$ & $7 \pm 1^{*}$ \\
Sympathetic BRS, & $-6.1 \pm 0.4$ & $-6.7 \pm 0.5$ & $-7.8 \pm 0.9$ \\
AU/beat/mmHg & & & \\
$\Delta$ SBP decreasing, $\mathrm{mmHg}$ & $-17 \pm 1$ & $-17 \pm 1$ & $-35 \pm 5^{*}$ \\
$\Delta$ DBP decreasing, $\mathrm{mmHg}$ & $-17 \pm 1$ & $-15 \pm 1$ & $-18 \pm 1$ \\
$\Delta$ MAP decreasing, $\mathrm{mmHg}$ & $-21 \pm 1$ & $-19 \pm 1^{\dagger}$ & $-26 \pm 2$ \\
$\Delta$ PP, mmHg & $0.1 \pm 0.6$ & $-1.0 \pm 1.2$ & $-16.0 \pm 3.6^{*}$ \\
$\Delta$ Heart rate, bpm & $24 \pm 3$ & $19 \pm 3$ & $10 \pm 1^{*}$ \\
\hline
\end{tabular}

Data are mean \pm standard error. BRS, baroreflex sensitivity; SBP, systolic blood pressure; $D B P$, diastolic blood pressure; MAP, mean arterial pressure; $P P$, pulse pressure. ${ }^{*} p<0.05$ vs. young women and young men; ${ }^{\dagger} p<0.05$ vs. young women and older women.

\section{RESULTS}

\section{BASELINE SUBJECT CHARACTERISTICS}

Table 1 shows the baseline demographics for the three subject groups. Body mass index, HR, and DBP were similar among the three groups. Young women and young men had similar SBP, MAP, $\mathrm{PP}$, cardiac, and sympathetic BRS. Older women had significantly higher MAP, PP, and lower cardiac BRS compared to the younger groups. Sympathetic BRS in the older women was similar to that observed in the young subjects (Table 2). As expected, MSNA BI was similar between young women and young men, but higher in older women (Table 1).

\section{BLOOD PRESSURE TRANSIENTS DURING BRS TESTS}

During the vasoactive drug bolus of nitroprusside, young women and young men had similar changes in SBP and PP while older women had significantly greater decreases in SBP and PP (Table 2). The decrease in DBP was similar between young women, young men, and older women (Table 2). This change in BP is further illustrated in Figure 2 as well. The decrease in MAP was similar between young women and older women, while young men had significantly smaller decreases in MAP. In addition, the older women had a significantly smaller increase in HR during infusion of nitroprusside compared to young women and men (Table 2).

\section{RELATIONSHIP BETWEEN BLOOD PRESSURE AND CARDIAC BRS}

The cardiac BRS was positively associated with the decrease in SBP (Figure 1) in young men $(r=0.41$, slope $=0.72, p<0.05)$ and older women (Figure 1; $r=0.60$, slope $=0.12, p<0.05$ ). However, there was no significant relationship in young women (Figure 1). The cardiac BRS was also positively correlated with DBP and MAP in older women $(r=0.59, p=0.05 ; r=0.70$, $p=0.01$, respectively), but not with the change in $\mathrm{PP}(r=0.43$, $p=0.12$ ). Cardiac BRS was not significantly associated with the decrease in DBP, MAP, or PP in young women $(r=0.09, p=0.58$; $r=0.12, p=0.45 ; r=-0.17, p=0.29$ respectively) or young men $(r=0.18, p=0.31 ; r=0.18, p=0.32 ; r=0.28, p=0.11$ respectively).

\section{RELATIONSHIP BETWEEN BLOOD PRESSURE AND SYMPATHETIC BRS}

Analysis of the relationship between the decrease in BPs and sympathetic BRS was performed for each subject group. No significant relationships were found for any of the subject groups.

\section{DISCUSSION}

The major new findings of the present study were (1) the magnitude of change in SBP during a vasoactive drug bolus was associated with cardiac BRS in young men and older women; and (2) these relationships were absent in young women. Thus, young men and older women with low cardiac BRS were more likely to have larger drops in SBP in response to nitroprusside, suggesting a more prominent role for cardiac (as opposed to sympathetic) $\mathrm{BRS}$ in responding to acute decreases in BP in these groups. The fact that these variables are not correlated in young women suggest that the baroreflex may be less sensitive to the drop in BP and also implies that the baroreflex response may be more complicated in this group, and are consistent with recent evidence that the female sex hormones influence baroreflex control mechanisms (Convertino, 1998; Shoemaker et al., 2001).

In response to input from baroreceptor afferents, neurons in the nucleus tractus solitarius (NTS) alter central autonomic output, ultimately controlling postganglionic sympathetic neural activity and vagal control of HR (Benarroch, 2008). Although cardiac and vascular sympathetic baroreflex responses are part of an integrated physiological response to changes in BP, they are not a single unit, and can be regulated independently of each other. Our group has previously shown that cardiac and sympathetic BRS are not correlated with each other in young men and young women (Dutoit et al., 2010), showing that changes in one arm of the reflex should not be assumed to reflect changes in the other. This is consistent with data from humans (O'Leary et al., 2003, 2005) and also animals, where HR and vascular resistance were shown to be differentially regulated in rabbits (Guo et al., 1982).

With regard to differences between the sexes, our present finding of similar cardiac and sympathetic BRS values between men and women is in agreement with several previous studies (Tank 


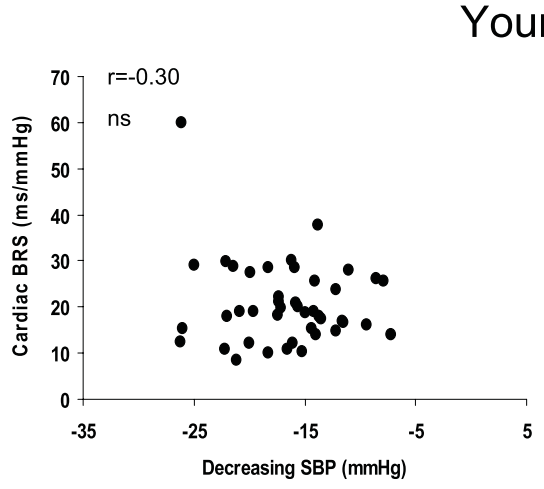

\section{Young Women}
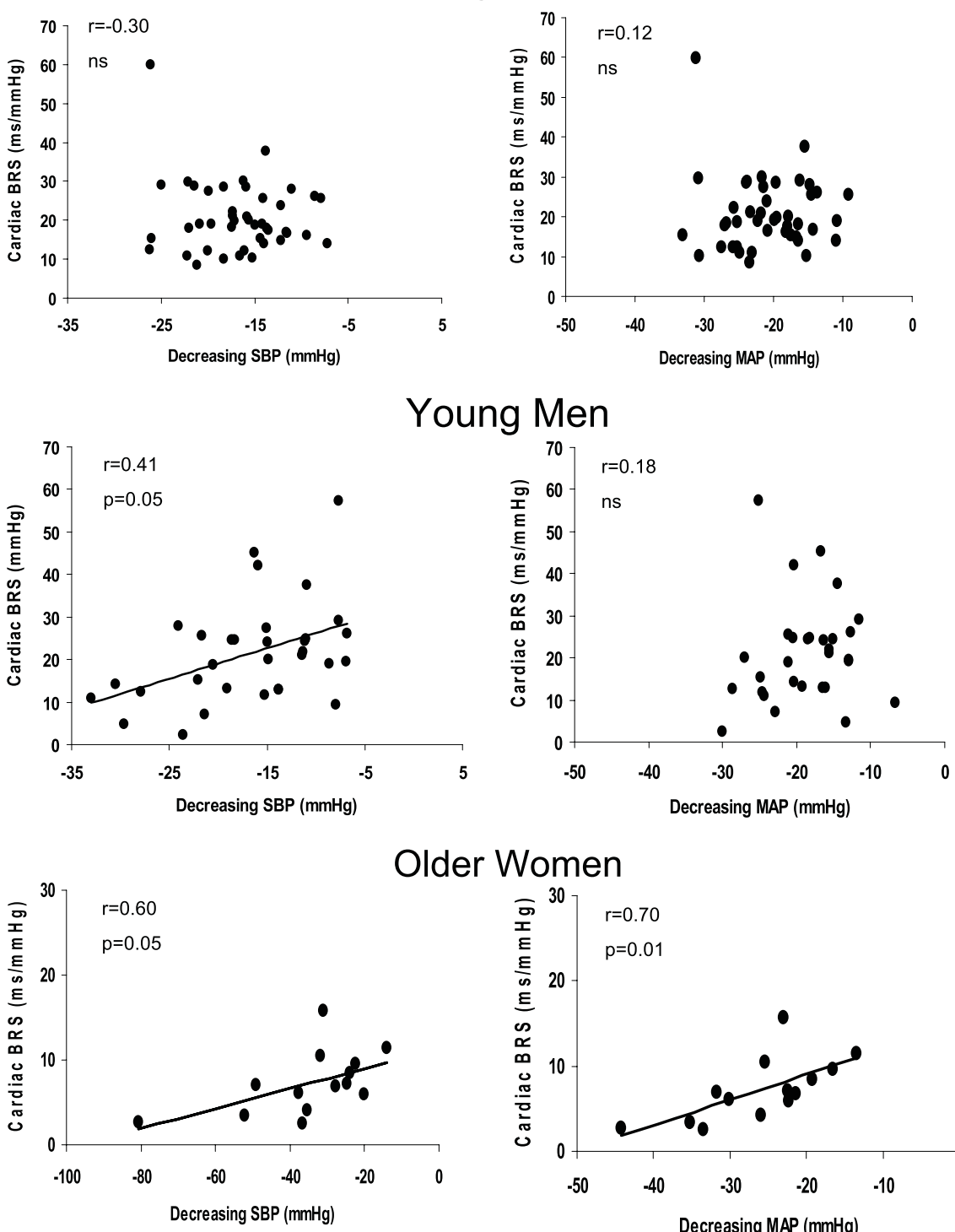

FIGURE 1 | Linear regression analysis of the correlation between the magnitude of decrease in SBP and cardiac BRS (left panel) and the magnitude of decrease in MAP during the decreasing pressure transients and cardiac BRS (right panel) in young women, young decreasing SBP and cardiac BRS was 0.72 and 0.12 respectively. The slope for the decrease in MAP and cardiac BRS in the older women was 0.30

et al., 2005; Dutoit et al., 2010). We did not observe any differences in baseline MSNA between young men and women, which is in contrast to the report from Christou et al. (2005) who also demonstrated a lower cardiovagal BRS, in response to incremental doses of phenylephrine in women compared to men. These differences may be due to the method of BRS testing because we examined both cardiovagal and sympathetic BRS responses over a wide range of pressures using nitroprusside/phenylephrine whereas Christou et al. reported cardiovagal responsiveness to increasing pressures to phenylephrine only.

Although young women and men demonstrated similar overall average BP transients and comparable mean cardiac and sympathetic BRS, the relationships we report in the present study

suggest that those averaged variables do not tell the whole "story." As in recent work from our group and others (Convertino, 1998; Hart et al., 2009a,b; Wallin et al., 2010), the evaluation of interindividual differences in BP transients and BRS values provided important additional insight in the present work. In particular, the relationships between individual values for cardiac BRS and $\triangle \mathrm{SBP}$ we observed in men, but not in young women, suggest an uncoupling between cardiac BRS and changes in BP that was specific to young women.

Our present findings are therefore consistent with recent data from our laboratory emphasizing sex-specific differences in BP regulation and the importance of sex hormones in the relationships between autonomic responses and systemic 

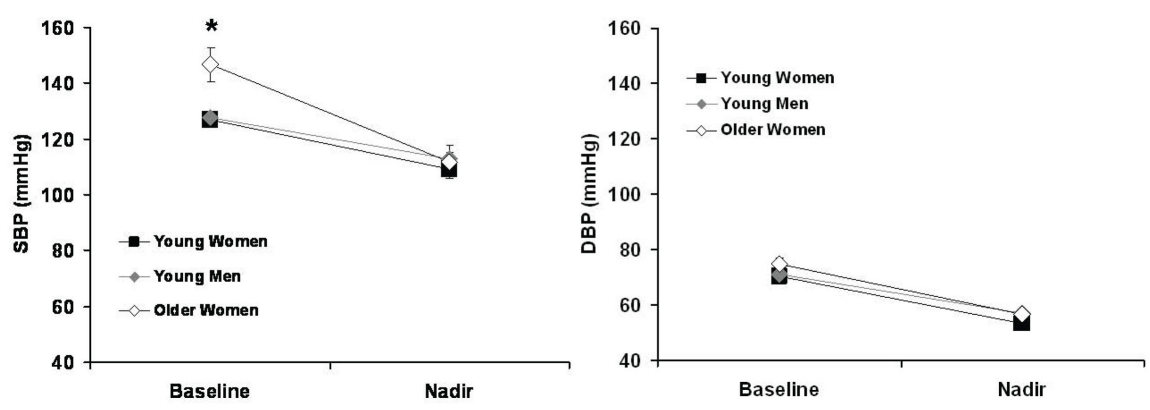

FIGURE 2 | Baseline and nadir SBP and DBP of young women, young men, and older women. ${ }^{*} p<0.05$ vs. young women and young men.

hemodynamics (Hart et al., 2009a, 2011, 2012). There is evidence that women have less effective BP regulatory mechanisms in response to acute challenges (Convertino, 1998; Barnett et al., 1999) and a reduced baroreflex buffering mechanism compared with young men (Christou et al., 2005). Moreover, the lack of a significant correlation in young women may be related to the sex-related differences in the sympatho-hemodynamic balance controlling BP (Hart and Charkoudian, 2011). For example, in contrast to young men and older women, there is no association between baseline MSNA and total peripheral resistance in young women. Therefore, for a given increase in sympathetic activity, there is less of a net effect of vasoconstriction and an attenuated BP response (Hart et al., 2011). Despite having similar sympathetic MSNA values, if women have less $\alpha$-adrenergic sensitivity and/or higher $\beta$-adrenergic sensitivity, then the net change in $\mathrm{BP}$ will be lower for a given change in MSNA will be less than men. However, Fu et al. (2009) demonstrated that MSNA, norepinephrine concentrations, and total peripheral resistance were similar between men and women during an orthostatic challenge. Furthermore, sexrelated differences in baroreflex buffering mechanisms were not related to differences in resting $\mathrm{BP}$, tonic sympathoadrenal activity, or any other baseline subject characteristic (Christou et al., 2005). In other words, interpreting the stimulus-response relationships in young women is more complicated and not all mechanisms are known.

Interestingly, young men and older women have comparable associations between SBP transients and cardiac BRS, even though older women showed significantly lower cardiac BRS and a greater magnitude of decrease in BP. This suggests that our results are not simply an effect of sex or aging, but related to the presence of sex hormones. All of the older women in this study were at least 1 year postmenopausal. Unfortunately, we do not have circulating estradiol or progesterone concentrations in all of our subjects, so it is difficult to determine if specific relationships exist for those hormones.

Our study has several limitations, first, we used a standard $(100 \mu \mathrm{g})$ dose of nitroprusside and the females in our study were generally smaller than the males, however this does not explain the differences in $\triangle \mathrm{BP}$ between young women and older women. In addition, body mass was not correlated with any of the BP transients (as we would expect smaller people to receive a greater relative dose and have a larger decrease in $\mathrm{BP}$ ) and correcting for body mass did not alter the significant associations reported in this study. A second limitation is that we did not study older men. We were interested in the potential influence of sex hormones on baroreflex regulation and compared young females to both older females, and young men who were included to serve as age-matched controls. The fact that older women and young men demonstrated similar associations despite the age-related changes in vascular function and BRS may have affected our interpretations. An additional limitation is that we calculated the magnitude of decrease in BP and compared to the BRS over the decreasing and increasing phases of a modified Oxford. However, if we only used decreasing pressure to calculate BRS, we would have to exclude subjects without enough points to perform linear regression and this would most likely be the subjects with the smallest changes in pressure.

In the present study, we found that cardiac BRS, but not sympathetic BRS, was related to the reduction in SBP during a hypotensive challenge using nitroprusside in young men and older postmenopausal women. This is of particular importance when considering BP regulation in older women who demonstrated a greater decrease in SBP and a lower cardiac BRS compared with young men and young women. Our data suggest that older postmenopausal women may be more dependent on HR (instead of sympathetic nerve activity) in maintaining BP during hypotensive stimuli. Furthermore, age and potential sex hormone-related declines in cardiac BRS might explain why the incidence of syncope increases in this group (Grossman et al., 2005). High resting MSNA in older women may minimize the ability to evoke sympathetically mediated vasoconstriction in response to acute hypotension (Cooper and Hainsworth, 2002). It is important to note, however, that we examined baroreflex control of BP in healthy men and women; therefore, we cannot make inferences about what these data may mean for individuals with cardiovascular disease or recurrent syncope.

In conclusion, cardiac BRS was associated with the magnitude of decrease in BP in young men and older women in response to a vasoactive drug bolus. Thus, young men and older women with lower cardiac BRS showed larger drops in BP in response to nitroprusside. Our results suggest a more prominent role for cardiac (as opposed to sympathetic) BRS in responding to rapid BP changes in young men and older women. The fact that these relationships do not exist in young women is consistent with other recent evidence that the female sex hormones influence baroreflex control of BP in humans. 


\section{ACKNOWLEDGMENTS}

The authors would like to thank the subjects for their participation in these studies. In addition, the authors thank the following

\section{REFERENCES}

Barnett, S. R., Morin, R. J., Kiely, D. K., Gagnon, M., Azhar, G., Knight, E. L., Nelson, J. C., and Lipsitz, L. A. (1999). Effects of age and gender on autonomic control of blood pressure dynamics. Hypertension 33, 1195-1200.

Benarroch, E. E. (2008). The arterial baroreflex: functional organization and involvement in neurologic disease. Neurology 71, 1733-1738.

Charkoudian, N., Martin, E. A., Dinenno, F. A., Eisenach, J. H., Dietz, N. M., and Joyner, M. J. (2004). Influence of increased central venous pressure on baroreflex control of sympathetic activity in humans. Am. J. Physiol. Heart Circ. Physiol. 287, H1658-H1662.

Christou, D. D., Jones, P. P., Jordan, J., Diedrich, A., Robertson, D., and Seals, D. R. (2005). Women have lower tonic autonomic support of arterial blood pressure and less effective baroreflex buffering than men. Circulation 111, 494-498.

Convertino, V. A. (1998). Gender differences in autonomic functions associated with blood pressure regulation. Am. J. Physiol. 275, R1909-R1920.

Cooper, V. L., and Hainsworth, R. (2002). Effects of head-up tilting on baroreceptor control in subjects with different tolerances to orthostatic stress. Clin. Sci. 103, 221-226.

Dutoit, A. P., Hart, E. C., Charkoudian, N., Wallin, B. G., Curry, T. B., and Joyner, M. J. (2010). Cardiac baroreflex sensitivity is not correlated to sympathetic baroreflex sensitivity within healthy, young humans. Hypertension 56, 1118-1123.

Fu, Q., Okazaki, K., Shibata, S., Shook, R. P., Vangunday, T. B., Galbreath, M. M., Reelick, M. F., and Levine, B. D. (2009). Menstrual cycle effects on sympathetic neural responses to upright tilt. J. Physiol. (Lond.) 587, 2019-2031.

Grossman, S. A., Shapiro, N. I., Van Epp, S., Kohen, R., Arnold, R., Moore, R., Lee, L., Wolfe, R. E., and Lipsitz, L. A. (2005). Sex differences in the emergency department evaluation of elderly patients with syncope. J. Gerontol. A Biol. Sci. Med. Sci. 60, 1202-1205.

Guo, G. B., Thames, M. D., and Abboud, F. M. (1982). Differential baroreflex control of heart rate and vascular resistance in rabbits. Relative role of carotid, aortic, and cardiopulmonary baroreceptors. Circ. Res. 50, 554-565.

Halliwill, J. R. (2000). Segregated signal averaging of sympathetic baroreflex responses in humans. J. Appl. Physiol. 88, 767-773.

Hart, E. C., and Charkoudian, N. (2011). Sympathetic neural mechanisms in human blood pressure regulation. Curr. Hypertens. Rep. 13, 237-243.

Hart, E. C., Charkoudian, N., Wallin, B. G., Curry, T. B., Eisenach, J., and Joyner, M. J. (2011). Sex and ageing differences in resting arterial pressure regulation: the role of the beta-adrenergic receptors. J. Physiol. (Lond.) 589, 5285-5297.

Hart, E. C., Charkoudian, N., Wallin, B. G., Curry, T. B., Eisenach, J. H., and Joyner, M. J. (2009a). Sex differences in sympathetic neuralhemodynamic balance: implications for human blood pressure regulation. Hypertension 53, 571-576.

Hart, E. C., Joyner, M. J., Wallin, B. G., Johnson, C. P., Curry, T. B., Eisenach, J. H., and Charkoudian, N. (2009b). Age-related differences in the sympathetic-hemodynamic balance in men. Hypertension 54, 127-133. individuals: Shelly Roberts, John Eisenach, Madhuri Somarju, Jessie Sawyer, Nancy Meyer, and Shirley Kingsley-Berg for technical assistance.

Hart, E. C., Joyner, M. J., Wallin, B. G. and Charkoudian, N. (2012). Sex, ageing and blood pressure: gaining insights from the integrated balance of neural and haemodynamic factors. J. Physiol. 590, 2069-2079.

Johnson, M. S., Demarco, V. G., Heesch, C. M., Whaley-Connell, A. T., Schneider, R. I., Rehmer, N. T., Tilmon, R. D., Ferrario, C. M., and Sowers, J. R. (2011). Sex differences in baroreflex sensitivity, heart rate variability, and end organ damage in the TGR(mRen2)27 rat. Am. J. Physiol. Heart Circ. Physiol. 301, H1540H1550.

Kienbaum, P., Karlssonn, T., Sverrisdottir, Y. B., Elam, M., and Wallin, B. G. (2001). Two sites for modulation of human sympathetic activity by arterial baroreceptors? J. Physiol. (Lond.) 531, 861-869.

O'Leary, D. D., Kimmerly, D. S., Cechetto, A. D., and Shoemaker, J. K. (2003). Differential effect of head-up tilt on cardiovagal and sympathetic baroreflex sensitivity in humans. Exp. Physiol. 88, 769-774.

O'Leary, D. D., Steinback, C. D., Cechetto, A. D., Foell, B. T., Topolovec, J. C., Gelb, A. W., Cechetto, D. F., and Shoemaker, J. K. (2005). Relating druginduced changes in carotid artery mechanics to cardiovagal and sympathetic baroreflex control. Can. J. Physiol. Pharmacol. 83, 439-446.

Parker, P., Celler, B. G., Potter, E. K., and McCloskey, D. I. (1984). Vagal stimulation and cardiac slowing. J. Auton. Nerv. Syst. 11, 226-231.

Shoemaker, J. K., Hogeman, C. S., Khan, M., Kimmerly, D. S., and Sinoway, L. I. (2001). Gender affects sympathetic and hemodynamic response to postural stress. Am. J. Physiol. Heart Circ. Physiol. 281, H2028-H2035.

Sundlof, G., and Wallin, B. G. (1977). The variability of muscle nerve sympathetic activity in resting recumbent man. J. Physiol. (Lond.) 272 383-397.

Tank, J., Diedrich, A., Szczech, E., Luft, F. C., and Jordan, J. (2005). Baroreflex regulation of heart rate and sympamen. Hypertension 45, 1159-1164.

Wallin, B. G., Hart, E. C., Wehrwein, E. A., Charkoudian, N., and Joyner, M. J. (2010). Relationship between breathing and cardiovascular function at rest: sex-related differences. Acta Physiol. (Oxf.) 200, 193-200.

Conflict of Interest Statement: The authors declare that the research was conducted in the absence of any commercial or financial relationships that could be construed as a potential conflict of interest.

Received: 16 March 2012; accepted: 21 May 2012; published online: 07 June 2012.

Citation: Barnes JN, Matzek LJ, Charkoudian N, Joyner MJ, Curry TB and Hart EC (2012) Association of cardiac baroreflex sensitivity with blood pressure transients: influence of sex and menopausal status. Front. Physio. 3:187. doi: 10.3389/fphys.2012.00187

This article was submitted to Frontiers in Integrative Physiology, a specialty of Frontiers in Physiology.

Copyright (c) 2012 Barnes, Matzek, Charkoudian, Joyner, Curry and Hart. This is an open-access article distributed under the terms of the Creative Commons Attribution Non Commercial License, which permits non-commercial use, distribution, and reproduction in other forums, provided the original authors and source are credited. thetic vasomotor tone in women and 